\title{
The inflammatory response from stereotactic body proton therapy versus stereotactic body radiation therapy: implications from early stage non-small cell lung cancer
}

\author{
Xingzhe D. Li ${ }^{1}$, Charles B. Simone $\mathrm{II}^{1,2}$ \\ ${ }^{1}$ Department of Radiation Oncology, Memorial Sloan Kettering Cancer Center, New York, NY, USA; ${ }^{2}$ New York Proton Center, New York, NY, USA \\ Correspondence to: Charles B. Simone II, MD. New York Proton Center, 225 East 126th Street, New York, NY 10035, USA. \\ Email: csimone@nyproton.com. \\ Provenance: This is an invited article commissioned by the Section Editor Dr. Hsin-Hua Nien (Attending physician, Department of Radiation \\ Oncology, Cathay General Hospital, Taipei). \\ Comment on: Li Y, Dykstra M, Best TD, et al. Differential inflammatory response dynamics in normal lung following stereotactic body radiation \\ therapy with protons versus photons. Radiother Oncol 2019;136:169-75.
}

Submitted Oct 27, 2019. Accepted for publication Nov 05, 2019.

doi: $10.21037 /$ atm.2019.11.41

View this article at: http://dx.doi.org/10.21037/atm.2019.11.41

\section{Introduction}

Lung cancer is the second most common noncutaneous cancer and the deadliest cancer in the United States (US) with 228,150 estimated new cases in 2019 (1). About $87 \%$ of lung cancers diagnosed in the US are non-small cell lung cancer (NSCLC), which currently has a 5-year overall survival (OS) rate of $23 \%$ across all stages, ranging from $57 \%$ for stage I to $4 \%$ for stage IV diseases (2). Radiation therapy (RT) is heavily utilized in the treatment of NSCLC. In early stage NSCLC (ES-NSCLC), stereotactic body radiation therapy (SBRT) delivered with photon radiotherapy has been shown to have high a local control rate and low incidence of severe treatment related toxicities (3), making it a standard of care curative option especially in medically inoperable patients (4). In stage III or locally advanced NSCLC (LA-NSCLC), radiation is used in approximately $60 \%$ of cases, often as part of bimodality treatment in conjunction with chemotherapy or as part of trimodality treatment delivered concurrently with chemotherapy followed by surgical resection (2). In stage IV or metastatic NSCLC, radiation is being increasingly used to palliate symptomatic lung or distant metastases and in specific settings, such as oligometastatic NSCLC, may be utilized with curative intent to target both the primary tumor and all metastatic disease sites (5).

\section{Proton therapy (PT) in early stage NSCLC}

PT offers potential advantages over conventional photon RT because of the unique physical characteristics of the Bragg peak, where most of the proton radiation dose is deposited across a very narrow range of depth, reducing irradiation of normal tissues near the tumor (6). In NSCLC treatment, major organs at risk (OARs) include the esophagus, heart, spinal cord and the normal lung tissue, which is often radiation dose limiting. Radiation pneumonitis (RP), caused by inflammation of lung tissues following radiotherapy, is one of the most difficult radiation-related toxicities to manage and can be fatal in a subset of patients (7). The acute cascade of inflammation may lead to chronic parenchymal changes such as pulmonary fibrosis that can be debilitating clinically for the patients. Fibrosis can particularly have an impact on quality of life in patients treated with SBRT, especially since many are medically inoperable and have considerable preexisting lung diseases (7). It is expected that by better sparing OARs, PT for NSCLC can reduce treatment-related toxicities and allow for safer dose-escalation, which in turn may improve survival by reducing life-threatening side effects and improving local control, respectively $(6,8)$.

While PT is known to better spare OARs compared with photon therapy for ES-NSCLC (9), multiple studies 
have also reported promising clinical outcomes of $\mathrm{PT}$ in ES-NSCLC, with low incidence of toxicities such as RP. Investigators from Loma Linda reported that none of the 111 stage I NSCLC patients treated with PT had clinical RP requiring steroid therapy (10). A recent meta-analysis comparing hypofractionated particle beam therapy (including PT) to photon SBRT for ES-NSCLC showed significantly lower rates of Grade $\geq 3 \mathrm{RP}$ in patient treated with particle beam therapy (0.9\% vs. 3.4\%, $\mathrm{P}=0.001)$ (11). Furthermore, a recent National Cancer Database (NCDB) propensity score matched analysis compared outcomes of all NSCLC patients (stages I-IV) treated with proton versus photon radiation, showing a significant 5 -year OS advantage for PT (22\% vs. 16\%, P=0.025) (12).

Numerous studies have demonstrated that PT has higher relative biological effectiveness (RBE) than photon therapy, allowing for increased tumor killing. In the clinical setting, we routinely use $\mathrm{RBE}=1.1$ as a dose scaling factor to equate the effects of protons and photons. However, the actual RBE at the distal edge of the proton beam has been shown to be much higher than 1.1 , which may be beneficial for tumor control, and which may account, in part, for the exceptionally low local failure rate observed in a multicenter prospective trial of proton reirradiation for recurrent NSCLC (13). However, this increased RBE may also deposit more than expected effective irradiation dose to the normal tissue just beyond the tumor, which has the potential to increase normal tissue inflammation compared to photon radiation $(14,15)$. SBRT, which delivers ablative dose of radiation most commonly in 5 or fewer fractions, has already been shown to elicit a greater degree of normal lung tissue responses compared to conventionally fractionated RT (16). This raises the question that whether stereotactic proton radiation therapy (SBPT) could elicit similar or even more inflammatory response in lung tissues compared to SBRT. While clinical pneumonitis rates have consistently been reported to be low with PT (11), a better understanding of the inflammatory effects of PT could allow for further refinement of the therapeutic ratio of PT, especially since the inflammatory cascades can influence both normal tissue toxicities and tumor killing immune responses, with the latter thought to be increasingly important in the era of more frequent combinations of RT and immunotherapy for NSCLC (17).

\section{Differential inflammatory responses: PT vs. photon RT}

In the July 2019 issue of Radiotherapy and Oncology, Li and colleagues from Massachusetts General Hospital provided some answers to the question of whether there are differential inflammatory responses of SBPT $v s$. SBRT (18). The investigators reviewed an institutional series of patients $(n=23)$ with ES-NSCLC who were treated by SBPT between 2008 and 2017. They carefully matched the cases to patients with similar demographic and tumor characteristics treated with SBRT at the same institution during the same period. To evaluate the inflammatory response of irradiated normal lung tissue, they used a validated surrogate marker for lung inflammation. Specifically, they plotted the computed tomography (CT) density changes (unit: Hounsfield Unit, HU) in ipsilateral lung parenchyma outside of target tumor volume against dose received by corresponding voxels to generate a quantitative measure of CT density changes in normal lung with a unit of HU/Gy. The CT density changes at subsequent time points ( 3,6 and 12 months following RT) were also compared.

The authors found that compared to matched SBRT cases, SBPT cases had numerically but not statistically significantly higher CT density changes in lung parenchyma. Interestingly, they did show a statistical difference in the response kinetics of lung density changes, with SBPT cases more likely to mount an accelerated course of inflammatory response compared to SBRT. Specifically, it was found that lung tissue density changes peaked early in SBPT cases at 3 months post-RT then started to decrease or stay unchanged at subsequent time points. On the contrary, SBRT cases did not show as robust acute lung responses but continued to result in increases in lung densities at later time points.

Based on these findings, the authors concluded that SBPT may be able to elicit more acute inflammatory responses in lung compared to SBRT. They speculated that these differential lung inflammatory responses might not contribute to any clinical differences in symptomatic pulmonary toxicities ( $\mathrm{RP}$ or radiation fibrosis) given the overall low incidence of such events in ES-NSCLC treated with SBRT or SBPT, but that the more accelerated inflammatory response associated with SBPT might offer potentially better synergistic effect with immunotherapy, for example with immune checkpoint inhibitors (ICI).

\section{Comments on the study}

We commend the authors for a notable publication comparing the inflammatory responses in normal lung tissues after SBPT $v s$. SBRT for ES-NSCLC. Although 
a relatively limited sample size to be able to assess for differences between protons and photons, this cohort of 23 patients treated with SBPT is one of the largest to date in NSCLC delivering true SBPT in 5 or fewer fractions.

Overall, the matching cohorts appear to be quite balanced except for the SBPT group having fewer treatment fractions [all having 3 or 4 fractions except only 1 patient treated in 5 fractions, compared to the SBRT group where all patient had $4(\mathrm{n}=15)$ or $5(\mathrm{n}=8)$ fractions of treatment]. Usually, with fewer fractions of stereotactic treatment, the biologically effective dose (BED) of radiation increases if the total dose stays the same based. However, we calculated the BEDs based on the dose fractionation regimen provided in the supplemental material and found there was no statistically significant difference in BEDs, suggesting that the investigators did consider BED when pairing SBRT patients to SBPT patients. On the other hand, prior reports suggest SBRT treatments with 3 or fewer fractions have relatively minimal lung density changes between 3 to 6 months, whereas SBRT treatments in 4-5 fractions tend to have more lung inflammations at 6 months compared to at 3 months (19). Therefore, the difference in radiation fractionation between the SBPT and SBRT groups in this study could have contributed, in part, to the results of differential inflammatory responses at early versus late time points. Another potential explanation of the more significant early inflammation with SBPT is the higher RBE at the distal edge of Bragg peak.

The authors did not include CT scans after 12 months following radiotherapy completion in their analysis. This is likely due to prior studies showing that the radiation induced lung responses tend to stabilize without further significant changes after 12 months (20). Since the SPBT patients in this study showed a trend of decreasing lung inflammation at later time points (9 months), it would be of interest for continued assessment with longer follow-up of these patients to see if a more significant difference in lung parenchymal changes will develop.

Given the extremely low incidence of RP reported in multiple prior studies for hypofractionated PT for ESNSCLC (11) and the limited number of patients in the current analysis, a clinically significant difference in rates of RP in this series would not be expected. However, as the authors did not report toxicities in this study, the differential inflammatory responses identified in this study do not provide a direct correlation with clinically meaningful endpoints such as symptomatic RP. While lung density changes was analyzed as the sole surrogate for lung inflammation, recent studies have attempted to integrate machine learning and radiomics features to provide more powerful and precise estimate of risks of RP development $(21,22)$.

$\mathrm{RP}$ in LA-NSCLC happens at much higher rate, likely due to larger lung volumes irradiated and the use of concurrent chemotherapy (7). The authors referenced one study comparing PT vs. photon RT that showed numerically but not statistically significantly higher rates of RP in LANSCLC patients treated with PT as a supportive evidence of potentially higher inflammatory response elicited by PT (23). It is worth noting that the RP rates dropped from over $20 \%$ down to $0 \%$ in the PT group when comparing patients enrolled during the first approximately 3 years of the trial to the last 3 years of trial. In contrast, $\mathrm{RP}$ rates did not change significantly in the photon RT group, highlighting the importance of experience with newer treatment modalities, which may significantly affect treatment outcome and toxicities, masking any true existence or non-existence of biological effect differences between modalities.

Meanwhile, two recently published prospective trials utilizing glycolytic score from 18F-2-fluoro-2-deoxy-Dglucose (FDG) positron emission tomography/computed tomography (PET/CT) as a surrogate marker for global lung parenchymal inflammation showed less lung parenchymal inflammation with PT compared to photon RT or combined photon-proton RT in LA-NSCLC patients $(24,25)$. While those findings contrasts the conclusions from this study in ES-NSCLC, it is possible that conventionally fractionated PT for LA-NSCLC may cause much less global lung inflammation due to fewer low dose irradiation to the normal lungs as a result of the physical nature of the proton beam, whereas the normal lung immediately adjacent to the target volume treatment with SBPT for ES-NSCLC may cause more a robust and earlier onset of focal lung inflammation as a result of the hypofractionation and increased $\mathrm{RBE}$ at the distal edge of Bragg peak, underscoring the differences between global versus focal lung inflammation.

Finally, from a technical perspective, it is noteworthy that the SBPT treatments in this study were mainly delivered with passive scattering proton treatment (PS-PT) started over a decade ago. While PS-PT can significantly reduce the integral irradiation dose to the normal lungs compared with photons, it can deliver higher doses to focal areas immediately adjacent to the target volume, especially when accounting for proton beam uncertainties 
and imaging uncertainties of not having volumetric imaging with cone beam computed tomography (CBCT) that was not available for the patients treated in the current study. Next generation PT delivered with pencil beam scanning proton treatment (PBS-PT) is able to offer more conformal planning for high dose treatment such as SBPT (26). Furthermore, CBCT is now in clinical use for PT and can further reduce uncertainty involved in setup and treatment planning (27). With these new technical advances in PT, we must refine our understandings about the dynamics of inflammatory responses in lung tissues after PT, which is of the utmost importance for future attempts to synergize the inflammatory and immunogenic responses of PT with immunotherapy.

\section{Acknowledgments}

None.

\section{Footnote}

Conflicts of Interest: The authors have no conflicts of interest to declare.

Ethical Statement: The authors are accountable for all aspects of the work in ensuring that questions related to the accuracy or integrity of any part of the work are appropriately investigated and resolved.

\section{References}

1. Siegel RL, Miller KD, Jemal A. Cancer statistics, 2019. CA Cancer J Clin 2019;69:7-34.

2. Miller KD, Nogueira L, Mariotto AB, et al. Cancer treatment and survivorship statistics, 2019. CA Cancer J Clin 2019;69:363-85.

3. Simone CB 2nd, Dorsey JF. Additional data in the debate on stage I non-small cell lung cancer: surgery versus stereotactic ablative radiotherapy. Ann Transl Med 2015;3:172.

4. Videtic GMM, Donington J, Giuliani M, et al. Stereotactic body radiation therapy for early-stage non-small cell lung cancer: Executive Summary of an ASTRO Evidence-Based Guideline. Pract Radiat Oncol 2017;7:295-301.

5. Gomez DR, Tang C, Zhang J, et al. Local Consolidative Therapy Vs. Maintenance Therapy or Observation for Patients With Oligometastatic Non-Small-Cell Lung Cancer: Long-Term Results of a Multi-Institutional, Phase
II, Randomized Study. J Clin Oncol 2019;37:1558-65.

6. Simone CB 2nd, Rengan R. The use of proton therapy in the treatment of lung cancers. Cancer J 2014;20:427-32.

7. Simone CB 2nd. Thoracic Radiation Normal Tissue Injury. Semin Radiat Oncol 2017;27:370-7.

8. Liao Z, Simone CB 2nd. Particle therapy in non-small cell lung cancer. Transl Lung Cancer Res 2018;7:141-52.

9. Wink KCJ, Roelofs E, Simone CB 2nd, et al. Photons, protons or carbon ions for stage I non-small cell lung cancer - Results of the multicentric ROCOCO in silico study. Radiother Oncol 2018;128:139-46.

10. Bush DA, Cheek G, Zaheer S, et al. High-dose hypofractionated proton beam radiation therapy is safe and effective for central and peripheral early-stage non-small cell lung cancer: results of a 12-year experience at Loma Linda University Medical Center. Int J Radiat Oncol Biol Phys 2013;86:964-8.

11. Chi A, Chen H, Wen S, et al. Comparison of particle beam therapy and stereotactic body radiotherapy for early stage non-small cell lung cancer: A systematic review and hypothesis-generating meta-analysis. Radiother Oncol 2017;123:346-54.

12. Higgins KA, O'Connell K, Liu Y, et al. National Cancer Database Analysis of Proton Versus Photon Radiation Therapy in Non-Small Cell Lung Cancer. Int J Radiat Oncol Biol Phys 2017;97:128-37.

13. Chao HH, Berman AT, Simone CB 2nd, et al. MultiInstitutional Prospective Study of Reirradiation with Proton Beam Radiotherapy for Locoregionally Recurrent Non-Small Cell Lung Cancer. J Thorac Oncol 2017;12:281-92.

14. Cuaron JJ, Chang C, Lovelock M, et al. Exponential Increase in Relative Biological Effectiveness Along Distal Edge of a Proton Bragg Peak as Measured by Deoxyribonucleic Acid Double-Strand Breaks. Int J Radiat Oncol Biol Phys 2016;95:62-9.

15. Underwood TSA, Grassberger C, Bass R, et al. Asymptomatic Late-phase Radiographic Changes Among Chest-Wall Patients Are Associated With a Proton RBE Exceeding 1.1. Int J Radiat Oncol Biol Phys 2018;101:809-19.

16. Diot Q, Marks LB, Bentzen SM, et al. Comparison of radiation-induced normal lung tissue density changes for patients from multiple institutions receiving conventional or hypofractionated treatments. Int J Radiat Oncol Biol Phys 2014;89:626-32.

17. Simone CB 2nd, Burri SH, Heinzerling JH. Novel radiotherapy approaches for lung cancer: combining 
radiation therapy with targeted and immunotherapies. Transl Lung Cancer Res 2015;4:545-52.

18. Li Y, Dykstra M, Best TD, et al. Differential inflammatory response dynamics in normal lung following stereotactic body radiation therapy with protons versus photons. Radiother Oncol 2019;136:169-75.

19. Diot Q, Kavanagh B, Schefter T, et al. Regional normal lung tissue density changes in patients treated with stereotactic body radiation therapy for lung tumors. Int J Radiat Oncol Biol Phys 2012;84:1024-30.

20. Bernchou U, Schytte T, Bertelsen A, et al. Time evolution of regional CT density changes in normal lung after IMRT for NSCLC. Radiother Oncol 2013;109:89-94.

21. Cunliffe A, Armato SG 3rd, Castillo R, et al. Lung texture in serial thoracic computed tomography scans: correlation of radiomics-based features with radiation therapy dose and radiation pneumonitis development. Int J Radiat Oncol Biol Phys 2015;91:1048-56.

22. Valdes G, Solberg TD, Heskel M, et al. Using machine learning to predict radiation pneumonitis in patients with stage I non-small cell lung cancer treated with stereotactic body radiation therapy. Phys Med Biol 2016;61:6105-20.

23. Liao Z, Lee JJ, Komaki R, et al. Bayesian Adaptive Randomization Trial of Passive Scattering Proton Therapy and Intensity-Modulated Photon Radiotherapy for Locally Advanced Non-Small-Cell Lung Cancer. J Clin Oncol 2018;36:1813-22.

24. Rice SR, Saboury B, Houshmand S, et al. Quantification of global lung inflammation using volumetric 18F-FDG PET/CT parameters in locally advanced non-smallcell lung cancer patients treated with concurrent chemoradiotherapy: a comparison of photon and proton radiation therapy. Nucl Med Commun 2019;40:618-25.

25. Jahangiri P, Pournazari K, Torigian DA, et al. A prospective study of the feasibility of FDG-PET/CT imaging to quantify radiation-induced lung inflammation in locally advanced non-small cell lung cancer patients receiving proton or photon radiotherapy. Eur J Nucl Med Mol Imaging 2019;46:206-16.

26. Lin L, Kang M, Huang S, et al. Beam-specific planning target volumes incorporating 4D CT for pencil beam scanning proton therapy of thoracic tumors. J Applied Clinical Medical Physics 2015;16:5678.

27. Veiga C, Janssens G, Teng CL, et al. First Clinical Investigation of Cone Beam Computed Tomography and Deformable Registration for Adaptive Proton Therapy for Lung Cancer. Int J Radiat Oncol Biol Phys 2016;95:549-59.
Cite this article as: $\mathrm{Li} \mathrm{XD}$, Simone CB 2nd. The inflammatory response from stereotactic body proton therapy versus stereotactic body radiation therapy: implications from early stage non-small cell lung cancer. Ann Transl Med 2019;7(Suppl 8):S295. doi: 10.21037/atm.2019.11.41 